\title{
DE LA FORTUNA ESCÉNICA DE ROJAS ZORRILLA EN LOS TEATROS DE MADRID
}

\author{
LUCIANO GARCÍA LORENZO \\ Instituto de la Lengua Española, CSIC \\ A Teresa Julio, \\ por tantas horas con Rojas Zorrilla
}

\section{RESUMEN}

Siguiendo la labor de René Andioc y Mireille Coulon para el siglo XVIII y de otros estudiosos en épocas posteriores, entre los cuales el propio autor del artículo, se ofrecen en este trabajo los datos correspondientes a los estrenos de Francisco de Rojas Zorrilla en los teatros madrileños, desde principios del siglo XVIII hasta nuestros días. De la actividad de cada siglo se extraen una serie de conclusiones para un mejor conocimiento de la recepción del autor toledano y el puesto que le corresponde en la suerte en los escenarios de nuestros clásicos.

Palabras clave: Rojas Zorrilla, puestas en escena, siglos XVIII-XXI.

\section{ABSTRACT}

Following the steps of René Andioc's work on the 18th century, as well as those of others' on later centuries (among them, the author of this article), this essay offers the data belonging to the premieres of Francisco de Rojas Zorrilla in Madrid's theaters from the 18th century up until today. It reflects on a number of features specific of each epoch in order to better understand the critical reception of the famous Toledan playwright, as well as its place in the canon of his Golden Age peers.

Key Words: Rojas Zorrilla, staging, XVIII-XXI centuries.

En los últimos años han ido aumentando considerablemente los estudios sobre la recepción del teatro clásico español y tanto en lo que se refiere a las épocas más cercanas como a la presencia en los escenarios a partir del siglo XVIII. El trabajo recopilatorio de Andioc y Coulon ${ }^{1}$ fue definitivo para empezar a entender adecuadamente lo sucedido en ese siglo, como otros más parciales dedicados a las dos centurias siguientes e incluso a los primeros años de la presente son testimonios de la dedicación a que nos referimos. En las páginas siguientes, y en los momentos adecuados, iremos haciendo referencia a estos trabajos.

${ }^{1}$ René ANDIOC y Mireille Coulon, Cartelera teatral madrileña del siglo XVIII (17081808). Toulouse, Presses Universitaires du Mirail, 1996, 2 vols. 
Y ahora, con motivo de su centenario, buen momento es para dejar patente cuál ha sido la fortuna de Francisco de Rojas Zorrilla, aprovechando, además, que las nuevas ediciones de las obras de este autor, como asimismo su bibliografía crítica, pueden ayudar en algunos aspectos a poner la labor de Rojas en el lugar que le corresponde y de esa manera completar la ya realizada, y muy bien, por libros recién aparecidos ${ }^{2}$, por otros con no muchos años como el de Teresa Julio ${ }^{3}$ y estudios más específicos como los llevados a cabo por M. E. Arenas Cruz, Felipe Pedraza, Teresa Julio... ${ }^{4}$ Todo ello va a facilitar, repetimos, poder situar al autor toledano en la escena de los últimos trescientos años, de la misma manera que el centenario calderoniano del 2000 dejó el sendero muy abierto para el autor de La vida es sueño o los interesados en Lope vieron aparecer ese mismo año un volumen dedicado, por este camino, al Fénix ${ }^{5}$.

Nuestra intención, con estas líneas, es contribuir a ese propósito y para ello vamos a ofrecer algunos datos y una aproximación a ellos, con el fin de, por una parte, acumular la información pertinente y, por otra, de ir haciendo la adecuada interpretación. Ya de antemano, y como primer testimonio del trabajo que hemos llevado a cabo, dejar constancia de la necesidad de hacer para los siglos XIX y XX lo que Ada M. Coe y Andioc y Coulon hicieron para el XVIII, y ello a pesar de los esfuerzos de Nicholson B. Adams, John Varey y sus «Fuentes para la historia del teatro en Espa-

${ }^{2}$ Sobre todo, el magnífico de Rafael GonZález Cañal, Ubaldo Cerezo Rubio y Germán Vega García-Luengos, Bibliografía de Francisco de Rojas Zorrilla. Kassel, Edition Reichenberger, 2007. Remitimos a este volumen, sobre todo y en este caso, para todo lo relacionado con el problema de atribuciones, obras en colaboración, etc.

${ }^{3}$ La recepción dramática: aplicación al teatro de Rojas Zorrilla. Kassel, Reichenberger, 1996.

${ }_{4}$ María Elena ARENAS CRUZ, «Las representaciones De Rojas en el siglo XVIII y su valoración en el Memorial literario», en Francisco de Rojas Zorrilla, poeta dramático. Actas de las XXII Jornadas de teatro clásico de Almagro. Ed. de Felipe B. Pedraza Jiménez, Rafael González Cañal y Elena Marcello. Almagro, Festival de Almagro-Universidad de Castilla-La Mancha, 2000, pp. 379-393; Felipe B. Pedraza Jiménez, «Abrir el ojo de Rojas Zorrilla bajo el antiguo régimen», en Criticón, 87-88-89, 2003, pp. 637648; Teresa Julio, «La suerte escénica de Entre bobos anda el juego», en La maravilla escrita, Antonio de Torquemada y el Siglo de Oro. Coordinadación de Juan Matas Caballero y José Manuel Trabado Cabado. Universidad de León, 2005, pp. 457-471. También, de la misma autora, «Rojas Zorrilla y su atractivo mediático», en Teatro, prensa y nuevas tecnologías. Madrid, UNED-Visor Libros, 2004, pp. 373-386.

${ }^{5}$ Sobre todo, el volumen Calderón en escena. Siglo XX. Ed. de José María Díez Borque y Andrés Peláez Martín. Madrid, Consejería de Cultura de la Comunidad de Madrid, 2000; también Luciano GARCía LORENZO y Manuel MuÑOZ CARABANTES, «El teatro de Calderón en la escena española (1939-1989)», en Estado actual de los estudios calderonianos. Kassel, Reichenberger-Festival de Almagro, 2000, pp. 351-382. Por lo que se refiere a Lope: Enrique GARCíA SANTO-TOMÁs, La creación del "Fénix». Recepción crítica y formación canónica del teatro de Lope de Vega. Madrid, Gredos, 2000 . 
ña», Irene Vallejo y Pedro Ojeda, los dos «Cuadernos bibliográficos» consagrados a los años 1930 a 1949, el artículo de Carmen Menéndez Onrubia dedicado a los comprendidos entre 1875 y 1900 (con listado para 18871899), la recogida de datos de Dougherty y Vilches para parte del primer tercio del siglo XX, la tesis de Muñoz Carabantes dedicada a los años 19391980, los diversos a la etapa posterior de Luciano García Lorenzo, y otras aportaciones que el lector puede encontrar en la bibliografía del volumen 22 de Cuadernos de teatro clásico $^{6}$. En fin, para la presencia de Rojas en el XVII, remitimos al excelente resumen de Teresa Julio en el trabajo ya citado dedicado a Entre bobos... y donde esta profesora señala adecuadamente la dificultad de hacer una «cartelera» de este siglo ${ }^{7}$.

${ }^{6}$ Nicholson B. AdAMS, «Siglo de Oro Plays in Madrid, 1820-1850», Hispanic Review, IV, 1936, pp. 342-357; Irene VALlEJo y Pedro OJEDA, El teatro en Madrid a mediados del siglo XIX. Cartelera teatral (1854-1864). Universidad de Valladolid, 2002; Cartelera teatral madrileña I: años 1830-1839. Madrid, C.S.I.C., 1961; Félix HerRero SAlgAdo, Cartelera teatral madrileña: II: años 1840-1849. Madrid, CSIC, 1963; varias carteleras teatrales de los años sesenta en la revista Segismundo, por Juan María DíEz TABOAdA y Luciano GARCía LOREnZO; Carmen MEnÉndez OnRUBIA, «El teatro clásico durante la Restauración y la Regencia», en Cuadernos de teatro clásico («Clasicos después de los clásicos»), 1990, pp. 187-208; Dru Dougherty y María Francisca VilCHES DE FRUTOS, La escena madrileña entre 1918 y 1926. Análisis y documentación. Madrid, Editorial Fundamentos, 1990; María Francisca VilCHES DE FRUTOS y Dru DOUGHERTY, La escena madrileña entre 1926 y 1931: un lustro de transición. Madrid, Editorial Fundamentos, 1997; Manuel MuÑoz CARABANTES, Puesta en escena y recepción del teatro clásico y medieval en España (1939-1989). Tesis presentada en la Universidad Complutense de Madrid, en 1992, y dirigida por Luciano Garcia Lorenzo. El volumen de Cuadernos de teatro clásico es el 22, 2006, y en él hay dos amplios trabajos de Javier Huerta Calvo y Héctor Urzáiz, con dos magníficos apartados bibliográficos.

${ }^{7}$ Hay, naturalmente, trabajos dedicados a otras ciudades de España: Narciso ALONSO CORTÉS, «El teatro en Valladolid», en BRAE, VIII, 1921, pp. 5-39 y 571-584; A. Par, «Representaciones teatrales en Barcelona durante el siglo XVIII», en BRAE, XVI, 1929, pp. 326-346, 492-513 y 594-614; J. MONTERo DE LA PUENTE, «El teatro en Toledo durante el siglo XVIII», en RFE, XXVI, 1942, pp. 187-208; Jean SENTAURENS, Séville et le théâtre. De la fin du Moyen Age à la fin de XVIIe siècle. Lille-Bordeaux, Presses Universitaires, 1994; F. DomíngueZ MATiTo, El teatro en La Rioja (1580-1808). Los patios de comedias de Logroño y Calahorra. Estudio y documentos. Logroño, Universidad de La Rioja, 1988; Germán VEGA GARCíA-LuENGOS, «El teatro barroco en los escenarios y en las prensas de Valladolid durante el siglo XVIII», en Teatro del Siglo de Oro. Homenaje a Alberto Navarro Gonzalez. Kassel, Reichenberger, 1990, pp. 639-673; María Teresa SUERO ROCA, El teatre representat a Barcelona de 1800 a 1830. Barcelona Institut del Teatre, 1987-1997, 4 vols.; J. M. SALA VALLDAURA, Cartellera del teatre a Barcelona (1790-1799). Barcelona, Publicacions de l'Abadia de Montserrat, 1990. El profesor José Romera Castillo lleva dirigiendo, desde la Universidad Nacional a Distancia, muchos años tesis doctorales de diferentes ciudades españolas, pero no pocas se encuentran sin publicar. 


\section{SIGLO XVIII}

Ofrecemos a continuación los datos que recogieron en su momento Andioc y Coulon, siguiendo un criterio alfabético para la presentación de las obras y respetando la fecha de 1805 que ellos se pusieron como límite. No damos los datos cronológicamente y a través de las representaciones porque no lo han hecho los trabajos existentes, tanto para el siglo XVIII como para el XIX, y lo único que haríamos sería complicar las cosas. Además, lo que nosotros pretendemos en estas páginas está más en consonancia con el criterio adoptado. Damos las títulos tal como Andioc y Coulon lo hicieron y también la autoría de los textos o las dudas que manifestaban. No ofrecemos la cifra exacta de algunos montajes porque tampoco estamos seguros de que no pocos datos se refieran a reposiciones y no a nuevas puestas en escena. Por supuesto, la primera y acertada aproximación a Rojas en el XVIII fue llevada a cabo por Arenas en 1999; repetimos los títulos y las cantidades de representaciones, pero ofrecemos los años y los teatros donde se pudieron ver estas funciones. Creemos que tener en este trabajo todos los elementos juntos, y al lado de los siglos posteriores, puede ayudar al lector que quiera acercarse al tema.

Los áspides de Cleopatra. Se representó de 1709 a 1798 en 47 ocasiones, sobre todo en las décadas treinta y sesenta. Lo hizo principalmente en el Teatro de la Cruz.

Los bandos de Verona. Se hicieron 36 montajes desde 1709 a 1705, sobre todo en las décadas de los diez y de los treinta. Teatro de la Cruz, principalmente.

El Caín de Cataluña. En 42 ocasiones, de 1708 a 1791. Fueron más numerosas las representaciones en el Teatro de la Cruz, pero también bastantes en el Príncipe.

Casarse por vengarse. En 23 ocasiones, pero en la segunda mitad de siglo, a partir de 1745. Casi todas en Teatro de la Cruz.

El Catalán Serrallonga y bandos de Barcelona. ¿De Rojas, Coello y Vélez de Guevara, se preguntan Andioc y Coulon? En 39 ocasiones y casi todas en el teatro de la Cruz.

Como la luna creciente también tiene el sol menguante. Andioc y Coulon se preguntan si es la obra de Vélez, Rojas Zorrilla y otro o la refundición de Hoz y Mota. 13 veces, sobre todo en el de la Cruz.

La confesión de un retrato. ¿Es la de Rojas o la de Francisco de Medina? Una sola vez, en 1709, en el Príncipe.

Del rey abajo, ninguno, García del Castañar. En 44 ocasiones, de 1710 a 1807. Con gran diferencia en el Teatro de la Cruz; la última en los Caños del Peral.

Donde hay agravio no hay celos, el amo criado. De 1708 a 1805 y en 117 ocasiones. En el Teatro de la Cruz la mayor parte.

Los encantos de Medea. Sólo en 2 ocasiones y en el de la Cruz: 1722 y 1735.

Entre bobos anda el juego (y todos van fulleros), Don Lucas del Cigarral. En 42 ocasiones, de 1715 a 1800 . Muy repetidamente en los años sesenta. También, fundamentalmente, Teatro de la Cruz.

Lo que quería ver el Marqués de Villena. Una sola vez, en 1710, y en el de la Cruz.

El más propio verdugo por la más justa venganza. De Rojas, Vélez y Coello. En 10 ocasiones, en la primera mitad de siglo (1710 a 1748) y en el de la Cruz. 
El mejor amigo y el muerto y evoción de las ánimas. ¿De Belmonte, Rojas y Calderón? ¿La de tres ingenios?, se preguntan Andioc y Coulon. De 1708 a 1736 en 16 ocasiones y, sobre todo, el día 1 de noviembre casi todos los años.

La más hidalga hermosura (y conde Fernán González). De Zabaleta, Rojas y Calderón. En torno a 50 montajes, de 1708 a 1805, y en Teatro de la Cruz, pero muchas también en el Príncipe.

El Monstruo de la Fortuna, y lavandera de Nápoles (Felipa Catanea). Andioc y Coulon se preguntan si es de Calderón, Montalbán y Rojas o de Rojas, Coello y Luis Vélez. 84 veces, de 1708 a 1807, sobre todo en el Teatro de la Cruz, pero también bastantes en el Príncipe.

No hay amigo para amigo. Una sola vez, en 1719, en el Príncipe.

No hay ser padre sino rey. En una ocasión y en el Teatro de la Cruz.

Obligados y ofendidos (o El gorrón de Salamanca). En 19 ocasiones, en la primera mitad de siglo (1708 a 1748), repartidas casi a partes iguales entre el de la Cruz y el Prínipe.

El pleito que tuvo el diablo con el cura de Madridejos. De Rojas, Vélez de Guevara y Mira de Amescua. En 15 ocasiones, de 1709 a 1767, y en el de la Cruz sobre todo.

Progne y Filomena. ¿De Guillén de Castro o de Rojas? En 27 ocasiones, de 1710 a 1776, principalmente en el de la Cruz, pero a partir de 1768 en el Príncipe. Sobre todo se ofreció en la década de los diez y de los sesenta.

Santa Isabel reina de Portugal. ¿De Rojas o Manuel Villaflor? De 1711 y sólo hasta 1737, nueve veces, la mayoría en el Príncipe.

Santa Táez. De Fernando de Zárate o Rojas. Sólo tres puestas en escena: 1713, 1713 y 1733. En el Príncipe todas ellas.

También la afrenta es veneno. De Rojas, Antonio Coello y Vélez de Guevara. 14 veces, de 1710 a 1806, pero fundamentalmente en la década de los treinta y los setenta. Se repartieron las representaciones entre los dos teatros.

Los trabajos de Tobías. De 1713 a 1783, en 14 ocasiones. Sobre todo en el de la Cruz.

La traición busca castigo. Sólo en 1736 y 1765 y las dos en el de la Cruz.

Las conclusiones esenciales a que podemos llegar para la presencia de Rojas en Madrid, completando los datos de Arenas y remitiendo a su estudio, que nosotros complementamos, son:

a) El teatro de Rojas permanece muy vivo en la escena del siglo XVIII. Son cientos de representaciones, exactamente 654, según Arenas, y decenas de obras las que se montan.

b) El texto más representado no es ninguno de los dos o tres que han quedado para el presente como canónicos en la lectura y en los escenarios; efectivamente, 117 veces pudo verse Donde hay agravios no hay celos, el amo criado. Lo hizo a lo largo de todo el siglo y parece que con una enorme aceptación. El segundo fue $E l$ Monstruo de la fortuna, y lavandera de Nápoles, con más de 80 puestas en escena y también a lo largo de todo el siglo. Poco más de la mitad de esta última se representaron Del rey abajo, ninguno y Entre bobos anda el juego, que incluso se pudieron ver menos 
que Los áspides de Cleopatra, El Caín de Cataluña y La más hidalga hermosura ${ }^{8}$.

c) Los textos que menos llegaron a los escenarios fueron: La confesión de un retrato, Lo que quería ver el Marqués de Villena y No hay amigo para amigo (un montaje) y Los encantos de Medea, La traición busca castigo (dos montajes).

d) Con mucha diferencia, es el Teatro de la Cruz el que acoge más puestas en escena y a mucha distancia el del Príncipe, aunque algunos textos se reparten a lo largo de los años en los dos locales.

e) Unas cuantas obras se representaron sólo en la primera mitad del siglo. Es el primer síntoma de la enorme selección que va a traer consigo la centuria siguiente.

\section{SIGLO XIX}

Nos servimos para los datos referidos al XIX a los trabajos que mencionamos al principio de este estudio. Por supuesto, y como ya hemos dejado escrito, carecemos, desgraciadamente, de información adecuada para muchos años del siglo que ahora nos ocupa, por lo que, naturalmente, habrá saltos en los años que figuran a continuación. También está claro, por simple comparación con los años inmediatamente siguientes, que la información de Ada M. Coe para las primeras décadas del XIX es muy limitada. Sería importante tener completa toda esa información para ir viendo la evolución en la escena de Rojas y las diferentes obras, pero no creo que afectaran a las deducciones de carácter más general a las que llegaremos. Adams, y sus datos no eran completos tampoco, por supuesto, concluye que Rojas es el quinto autor más representado, después de Tirso, Calderón, Lope y Moreto. También, como para el siglo XVIII, seguimos el orden alfabético de los títulos de las obras y por las mismas razones que dimos antes.

Abre el ojo o aviso a los solteros. Hubo un montaje en el Príncipe en 1812, 1824 ocho representaciones, 1826 dos, 1827 una, 1828 una, 1829 tres, tres montajes en 1830 , cuatro en 1833, cuatro en 1834, cinco en 1838, una en 1839. En el teatro de la Cruz casi toda ellas.

El amo criado. Se ofreció en 1819, en el Teatro de la Cruz, 1821 y 1822 dos representaciones, 1829 tres, 1831 dos, 1832 dos días, 1833 también dos, 1845 dos, 1846

${ }^{8}$ Coincide sólo en parte con las elegidas, siete, por Bernardo de Iriarte en su cartainforme al Conde Aranda: Abrir el ojo, Entre bobos anda el juego, Casarse por vengarse, Sin honra no hay amistad, Lo que son mujeres, No hay amigo para amigo y No hay agravio donde no hay celos. Sólo seleccionará más obras de dos autores: Calderón y Moreto. Vid. Emilio PALACiOS FERnÁNDEZ, «El teatro barroco español en una carta de Bernardo de Iriarte al Conde Aranda (1767)», en Cuadernos de teatro clásico, 5, 1990, pp. 43-64. 
cuatro días, 1847 (cinco días en dos ocasiones), 1859. Se dieron en los Teatro de la Cruz y, sobre todo, Príncipe en la década de los treinta, Museo en los cuarenta y Variedades y Teatro Circo en los cincuenta.

Casarse por vengarse. En 1805 una representación, 1824 dos. Así ofrece los datos Adams.

El catalán Serrallonga y bandos de Cataluña (o Barcelona) o Los bandos de Cataluña, Catalán Serrallonga. En el Teatro de la Cruz, en 1813 y en 1815. Dos representaciones en 1822, cinco en 1830. En el Teatro del Príncipe.

Del rey abajo, ninguno, García del Castañar o El Labrador más honrado. Se hicieron montajes en 1810 (con el título No ha de permitir agravio, del Rei abaxo ninguno, en 1810) y en 1811; todos los años de 1820 a 1827 , con varias representaciones cada vez, incluso 9 en 1824; de 1830 tres días, 1831 dos días, 1832 (siete días), 1836 (cuatro días), 1837 en cuatro días, 1839 (cinco días), 1843, 1844 (dos días), 1945, 1946 once días en total, 1847 cinco días, 1848 un día, 1854, en dos ocasiones (tres días), 1855 en cuatro ocasiones (siete días), 1856, 1859 (dos días), 1862 en un montaje (cuatro días), 1887-88 (cuatro), 1888-89 (tres), 1892-93 (un día).. Los Teatros fueron Príncipe y Cruz, sobre todo, pero también Buena Vista, Circo y Variedades.

Los encantos de Medea. 1830, tres representaciones; también tres en 1831. Adams no indica el Teatro.

Entre bobos anda el juego o Don Lucas del Cigarral. Montajes en 1834 tres, 1835, 1855, 1856 en tres ocasiones (cuatro días), 1858, 1863 (cuatro días en una ocasión), 1864, cinco días en una ocasión en los primeros días del mes enero, pero es casi con seguridad el montaje con que se cerró 1863 y que también se vuelve a dar en tres ocasiones en los meses de enero, febrero y marzo. También en las temporadas 1887-88 (cinco representaciones), 1888-89 (cinco), 1889-90 (dos), 1890-91 (dos), 1891-92 (tres), 1895-96 (once), 1896-97 (seis) y 1897-98 un día), 1899 (zarzuela sobre la obra de Rojas). Se dan en el Teatro Príncipe en los treinta, en las décadas de los cincuenta y sesenta en el Circo y Variedades y en los ochenta en el Teatro Español.

El monstruo de la fortuna, la lavandera de Nápoles, Felipa Catanea. En 1821 tres representaciones, en 1822 una, en 1824, cuatro, en 1832 tres.

Lo que son mujeres. Puesta en escena en 1822 cuatro representaciones, 1825 dos, 1826 tres, 1827 dos, 1828 una, 1929 cinco, 1831 tres, 1833 en dos ocasiones (tres días), 1835 una, 1836 dos, 1837 una, 1938 una ocasión pero dos días, 1839 en tres ocasiones, 1840 dos, 1844 en dos ocasiones, 1846 tres veces con seis representaciones, 1847 y 1848 una cada año, 149 tres representaciones, 1850, una, 1854 una, 1859 en una ocasión con cuatro funciones. Los locales fueron Príncipe, Cruz, Tres Musas, Circo, Museo y Variedades.

Exponemos seguidamente las conclusiones que podríamos extraer de esta información, pero queremos dejar sentado que la falta de un trabajo metódico y riguroso de la labor teatral en el Madrid del XIX y, por supuesto, en las diferentes ciudades de España, dejan abierta la puerta para corregir — quizás- y, sobre todo, completar nuestras opiniones. La confianza que tenemos en la mayor parte de lo existente es relativa y de ahí que algunos datos que ofrecemos somos muy conscientes de que deben someterse a un contraste manifiesto. Naturalmente, sí que son válidos estos datos para las conclusiones muy precisas que queremos exponer y que son las siguientes: 
a) Frente a la aparición continua de prácticamente todos los títulos de Rojas en el siglo XVIII, en el XIX son bastante menos los textos que se llevan a la escena. Naturalmente, ello está en la línea de lo que sucede con todos los autores de nuestro teatro áureo, pues tampoco Tirso y Calderón, pero también Lope o Moreto, aparecen con la frecuencia que lo habían hecho antes. Los adeptos al teatro clásico tendrán oportunidades de ver algunas obras a lo largo de siglo, pero el repertorio es corto y no precisamente conformado en muchísimas ocasiones por los títulos que hoy reconocemos mejores.

b) Sin embargo, en el caso de Rojas será en este siglo cuando se va a llevar a cabo el proceso de canonización de su teatro y Entre bobos anda el juego y El labrador más honrado... van a quedar, desde este tiempo, ya reconocidas como las obras por excelencia del teatro del autor toledano, hasta tal punto que, si seguimos el trabajo de Menéndez Onrubia, en la décadas finales del XIX sólo se representan estas dos piezas de Rojas. Llaman la atención, por otra parte, dos textos que llegarán a los escenarios con frecuencia en ciertos momentos del XIX y cuya aceptación ya venía del siglo anterior; nos referimos a El amo criado y Lo que son mujeres, con un número de representaciones que contrasta con el olvido a que han llegado posteriormente.

c) Recordar la zarzuela titulada Don Lucas del Cigarral, refundición de Tomás Luceño y Carlos Fernández Shaw, con música de Amadeo Vives (1999). También de finales del XIX es la zarzuela Entre bobos anda el juego, libreto de A. M. Ballester y música del valenciano J. Valls Volart ${ }^{9}$.

d) Los montajes se llevan a cabo en buena parte del XIX a través, bien es sabido, de refundiciones. Rojas es un caso más y Félix Enciso Castrillón, Hartzenbusch, Eduardo Asquerino, Manuel Eduardo de Gorostiza y Tomás Luceño, lo harán con algunos textos ${ }^{10}$. También a recordar que muchas de las funciones se completaban con música y/o una pieza breve, fundamentalmente un sainete; Don Ramón de la Cruz y Juan Ignacio González del Castillo acompañarán con frecuencia el nombre de Rojas con ese tipo de obritas.

${ }^{9}$ En Apéndice ofrecemos las fichas correspondientes a la zarzuela de Vives y a las refundiciones.

${ }^{10}$ Un testimonio significativo ya lo ofreció David Gies: durante el periodo 18201833 Rojas es el cuarto autor clásico con más refundiciones (116) y 122 representaciones. Delante estarán Tirso, Lope, Calderón y después Moreto, con 82 refundiciones, aunque con más funciones (164). Vid. David T. GIES, «Notas sobre Grimaldi y el 'furor de refundir' en Madrid (1820-18339», en Cuadernos de teatro clásico, 5, 1990, pp. 111-124. Vid. también Charles GANELIN, Rewriting theatre: the "comedia» and the ninenteeth-century «refundición». Lewisburg, Bucknell University Press, 1994. 
e) También a señalar que, como creo dejamos claro en la exposición de datos y cifras, se ofrecen en muchos momentos los montajes durante varios días e incluso un mismo montaje se repone con frecuencia días después o a lo largo de unas pocas semanas, repitiéndose la pieza breve que le acompañó en su primera aparición.

f) Los teatros serán prácticamente todos los que funcionan regularmente en este tiempo. Siguen el Príncipe y el de la Cruz como albergues indiscutibles — sobre todo en las décadas primeras- incorporándose luego Rojas al repertorio principalmente del Teatro Circo y del Variedades. A destacar el éxito de la Compañía de María Guerrero y Fernando Díaz de Mendoza, a finales de siglo, en el Teatro Español de Madrid con Entre bobos anda el juego. Carmen Menéndez dedica adecuada atención a esto en el trabajo que publica en este mismo volumen.

\section{SIGLOS XX Y XXI}

¿Abre el ojo! Director: Fernando Fernán Gómez. Adaptación: José Manuel Caballero Bonald. Escenografía: Cristina Borondo. Figurines: Javier Artiñano. Intérpretes: Juan Diego, Charo Soriano, Francisco de Osca, Carmen Maura, Vicente Cuesta, Tina Sáinz, Maite Blasco, Pedro de Río, Ana Fríjola, Francisco Matute, Francisco Pérez, Alfonso Vallejo, Enrique Pérez, José Antonio Camacho. Lugar y fecha: Teatro María Guerrero, 15 de diciembre $1978^{11}$.

Amo y criado. Director: Ricardo Calvo. Adaptación de Tomás Luceño. Compañía Matilde Moreno y Ricardo Calvo. Lugar y fecha: Teatro Español, 18 de marzo $1919^{12}$.

Entre bobos anda el juego. Director: Cayetano Luca de Tena. Adaptación: José García Nieto. Escenografía y figurines: Emilio Burgos. Música: Manuel Parada de la Puente. Intérpretes: Compañía del Teatro Español, con Guillermo Marín, María Jesús Valdés, Gabriel Llopart, Cándida Losada, José Capilla, Rafael Bardem, Esperanza Grases, Alberto Bové, Antonio Riquelme. Lugar y fecha: Teatro Español, 6 de diciembre 1951.

Entre bobos anda el juego. Director: Agustín Magán. Intérpretes: Teatro de Cámara «Ditea» de Santiago de Compostela. Intérpretes: Mariluz Kiler, José Domingo Castaño, Juan Pérez. Lugar y fecha: Teatro Beatriz (Teatro nacional de cámara y ensayo), temporada 1965-66.

11 Vid., Natalie CAÑIZARes Boudrof, Memoria de un escenario. Teatro María Guerrero. (1885-2000). Madrid, INAEM, Centro de Documentación teatral, 2000, pp. 269 y ss.; César Oliva, La última escena (Teatro español de 1975 a nuestros días). Madrid, Cátedra, 2004, pp. 66-71.

12 Datos que recogemos de Dru Dougherty y María Francisca Vilches, La escena madrileña entre 1918 y 1926. Análisis y documentación, cit., p. 180. En la página 66, al resumir la temporada 1918-19 leemos, sin embargo: «El teatro clásico español apenas existía para el público madrileño durante 1918-19, limitándose a tres autores y cinco obras: P. CALDERÓN DE LA BARCA, con La vida es sueño y El alcalde Zalamea, LOPE DE VEGA, con La estrella de Sevilla y Las famosas asturianas (adaptada por Muñoz Seca), y L. VÉlez DE GuEVARA, con Reinar después de morir.» 
Entre bobos anda el juego. Director: Gerardo Malla. Adaptación: Rafael Pérez Sierra y Gerardo Malla. Esconografía y vestuario: Pedro Moreno. Música: Miguel Malla. Intérpretes: Comapañía nacional de teatro clásico, con Cristina Marcos, Mónica Cano, Fanfri Topera, Rafael Castejón, Francisco Lahoz, Jesús Fuente, Raafel Ramos de Castro, Jesús Castejón, Paloma Paso Jardiel. Lugar y fecha: 8 de julio 1999, Hospital de San Juan, Festival de Almagro; 12 de noviembre de 1999 en el Teatro de la Comedia de Madrid.

Garcia del Castañar. Compañía Margarita Xirgu y Enrique Borrás, Lugar y fecha: Teatro Español, 1 de diciembre $1933^{13}$.

Obligados y ofendidos. Director: Juan José Granda. Adaptación: Fernando Doménech. Música: Fernando P. Ruano. Escenografía y vestuario: Malgorzata Zak. Intérpretes: Compañía de la Real Escuela Superior de Arte Dramático de Madrid, con Carmen Caballero, Angel Velasco, Antonio Villa, Paco Hernández, Luis Felpeto, Iñaki Arana, Puchi Lagarde, Javier Manzanera, Goyo Pastor, Laura Domínguez, Tomás Repila, Noelia Tejerina, Sol Montoya. Lugar y fecha: 7 de julio 1998, Teatro Municipal (Festival de Almagro); 11 de septiembre 1998, Teatro de la Comedia de Madrid ${ }^{14}$.

\section{Fuera de Madrid se han estrenado los siguientes montajes de Rojas:}

¿Abre el ojo! Intérpretes: «Aula-Jácara», Instituto de Bachillerato Jaime I de Alicante. Lugar y fecha: Instituto de Formación profesional de Alicante, 1 abril 1984.

¿Abre el ojo! Intérpretes: «Agora Grup de teatre», Carcagente (Valencia). Lugar y fecha: XXII Festival de Hita (Guadalajara), 7 de julio 1986.

¿Abre el ojo! Dirección: Francisco Plaza. Versión: Emilio del Valle y Francisco Plaza. Escenografía e iluminación: Francisco Leal. Vestuario: Pedro Moreno. Intérpretes: Cristóbal Suárez, Amparo Marín, Chema de Miguel, Jorge Muñoz, Angeles Martín, Carolina Sola, Rosa Herrera, José Pedro Carrión, Paco Pepe Martínez, Chete Guzmán. Local y fecha: Patio de Fúcares (Festival de Almagro); 24 de julio 2002.

¿Abre el ojo! Director: Javier Mateo. Versión: Sofía Eiroa y Javier Mateo. Escenografía: Javier Mateo y Antonio Fernández. Vestuario: Loreto Suárez. Música: Antonio

${ }^{13}$ Escribe Gil Fombellida: «Prácticamente ignorado por la prensa, el 2 de diciembre de 1933 se pone en escena un segundo texto de Tirso de Molina, titulado El estudiante que se a va a acostar, representado, dentro de un programa doble, junto al drama de Francisco Rojas Zorrilla, García del Castañar. De entre los periódicos consultados, tan sólo dos brevísimas reseñas, bastante desfavorables, aluden a este montaje. En ellas se califica la representación de «balbuceo dramático», inadecuado para una función ordinaria del Español (El Liberal), y de «esbozo de teatro» sin interés escénico e insignificante para el público (El Debate). Tampoco la puesta en escena cumple, en este caso, con las expectativas de la crítica ante la recuperación de un texto clásico, resultando demasiado extravagante en el vestuario y en la interpretación...» «M. ${ }^{\mathrm{a}}$ Carmen GIL FombellidA, Rivas Cherif, Margarita Xirgu y el teatro de la II República. Madrid, Fundamentos, 2003, p. 248. Antonina Rodrigo (Margarita Xirgu. Madrid, Aguilar, 1988, p. 271) sólo da la noticia del esteno sin más.

${ }^{14}$ Este montaje estuvo también en el Festival de El Chamizal (El Paso, Estados Unidos), en marzo de 1999. En este Festival se han podido ver las siguientes puestas en escena de Rojas: Donde hay agravios no hay celos (1980), por «Teatristas de Aguascalientes», Aguascalientes, México; Entre bobos anda el juego (1985), por la Escuela de Bellas Artes de la Universidad Autónoma de Chihuahua, México; Abre el ojo (1989), por el Instituto mexicano del Seguro social, Monterrey, Nuevo León, México; Abre el ojo (2003), por «Producciones inconstantes», dirección de Francisco Plaza, Madrid, España. 
José Gómez. Intérpretes: Aula de teatro de la Universidad de Almería, con Francisco Pardo, David Sánchez, Vicente Corredera, Neila Díaz, Marina Cayuela, María del Carmen García, Ana Carmona, Natalia Puertas, Ricardo Sierra, David Cantos. Lugar y fecha: Teatro Apolo de Almería (Jornadas de teatro del Siglo de Oro), 21 de marzo 2003.

Abre el ojo. Director: Antonio Barrios. Escenografía: Estefanía B: Mejías. Intérpretes: «Teatro Par, Córdoba», con Alvaro Barrios, Belén Martínez, Jordi Aguilera, Ricardo Luna, Belén Orihuela, Carmen Rey, Angel Lajara, Esteban Jiménez. Lugar y fecha: 28 de febrero 2004; Teatro Garnelo, Montilla (Córdoba).

Donde hay agravio no hay celos. Director: Ignacio Sánchez Pascual. Versión: Luis García Araus. Intérpretes: Compañía ComicAstros, con Xavier Olza, Jorge Gurpegui, Javier Ortiz, Bruno Ciordia, Andrea Soto, Chema Ruiz, Ana Huete. Lugar y fecha: Claustro de San Pedro (Festival de teatro clásico), Olite (Navarra); 14 de agosto $2004^{15}$.

Entre bobos anda el juego. Director: César Oliva. Escenografía: Juan Antonio Molina. Vestuario: José A. Arnaldos. Música: José María Galiana. Intérpretes: Compañía Julián Romea, Teatro estable de Murcia, con Isabel Barceló, Resu Morales, Manolom Ortín, Paco Paredes, Juan Bastida, José A. Arnaldos, Paco Leal, Laura Navarrete, Angel Amorós. Lugar y fecha: Festival internacional del Mar Menor de San Javier (Murcia), 7 de agosto 1981. Se representó junto al entremés de Calderón El desafío de Juan Rana y también las canciones «Poderoso caballero es Don Dinero» y «Boda en el campo».

Entre bobos anda el juego. Intérpretes: «Corral de Calatrava». Lugar y fecha: Corral de Calatrava (Ciudad Real), 28 de marzo 1984.

Entre bobos anda el juego. Dirección y adaptación: Angel García Suárez. Escenografía y vestuario: Carlos Alberto Abad. Música: Luis Andrés Haro. Intérpretes: Aitana Sánchez Gijón, Cayetana Guillén Cuervo, Fernando Guillén Cuervo, Iñaki Miramón, Alberto Muyo, Manuel Muñoz Cuenca, Carlos Alberto Abad, Enrique Narro, Carlos Moreno, Angel García Suárez. Lugar y fecha: 22 de febrero 1991; Teatro Lope de Vega de Sevilla.

Entre bobos anda el juego. Intérpretes: Compañía «La Murga», Cartagena (Murcia), 1997.

Entre bobos anda el juego. Dirección: Arturo Castro. Adaptación: Arturo Castro y Francisco Prieto. Diseño de escenografía: Giovanni Palladio. Diseño de vestuario: Azucena Rico. Intérpretes: Compañía «Teatro margen», con Félix Corcuera, Carlota Cangas, Angeles Arenas, Carlos Casero, José Antonio Lobato, Aída Estrada, Borja Roces, José Luis San Martín. Lugar y fecha: Teatro Jovellanos, Gijón (Asturias; 18 diciebre 1998.

Entre bobos anda el juego. Dirección y versión: Ernesto de Diego. Escenografía: Javier Ruiz de Alegría. Vestuario: Ana Serrano. Intérpretes: Compañía «Corral de Comedias», con Covadonga Calderón, Nieves Carrión, Norton P., Vicente Nove, Nacho Sánchez, Antonio León, Almudena S. Pascual, Félix Espinosa. Lugar y fecha: Corral de Comedias, Almagro (Ciudad Real), 2006. Esta Compañía había llevado a cabo un primer montaje en 1998, montaje que se ha repuesto en otras ocasiones.

15 Vid. Felipe B. Pedraza Jiménez, Donde hay agravios no hay celos: un éxito olvidado, en la muy reciente publicación en la red Teatro de palabras. Revista sobre teatro, 1, 2007, pp. 155-184, donde estudia la suerte de esta obra, fundamentalmente en el siglo XIX con la atención a ella prestada por Hartzenbusch. 
Los tres blasones de España. Director: Ricardo Romanos (luego Miguel Munárriz). Intérpretes: Vecinos de Calahorra (La Rioja). Lugar y fecha: Plaza de la Catedral de Calahorra, desde el año 1999 y durante algunos días del mes de agosto. Se representa cada año una jornada de la obra.

Dado que no son muchos los años del siglo XXI, hemos preferido no diferenciarlo del XX. Tampoco, bien es cierto, nada especial por los resultados obtenidos nos obligaría a ello. Estas son las ideas fundamentales que podríamos deducir de los datos ofrecidos, pues es nuestro deseo dedicar próxima atención a algunos de los montajes que recogemos:

a) Pocos, muy pocos, los montajes dedicados a Rojas. En el primer tercio de siglo la pobreza, incluso, es extremadamente llamativa y escandalosamente pobre. Y no solo por lo que se refiere al toledano, sino al teatro clásico en general. Naturalmente, también mencionar que falta una recogida de datos adecuada para muchos años de ese primer tercio, lo cual parece se está paliando últimamente. A recordar en ese primer tercio de siglo un montaje por parte de la Xirgu —El labrador más honrado...-, bajo el título Garcia del Castañar, pero parece, por las reseñas de prensa, con escaso acierto.

b) Después de 1939, no se puso en escena a Rojas hasta 1951, en montaje de Cayetano Luca de Tena. Hubo que esperar a 1978, con ¡Abre el ojo! en versión de Nieva en el recién creado Centro dramático nacional, y al de César Oliva en los ochenta, con Entre bobos anda el juego, para que se abrieran más posibilidades y precisamente con estos textos. Efectivamente, llama la atención que sean en la práctica estos títulos los que se repiten continuamente y hasta las fecha más cercanas, y tanto por compañías profesionales como de carácter más vocacional. Puede que no sorprenda tanto con Entre bobos..., tiempo atrás convertida en referencia fundamental de Rojas, como ya mencionamos, pero sí con iAbre el ojo!, que, naturalmente, es elegida en varias ocasiones siguiendo el eco que tuvo el montaje de Fernando Fernán Gómez.

c) Rojas tardó en llegar trece años (1999) a la Compañía nacional de teatro clásico, pero ya está en su repertorio. No hay sorpresa en la elección -Entre bobos anda el juego- y en un montaje que se recordará no como uno de los mejores de la CNTC, pero que gustó y divirtió al público ${ }^{16}$.

d) Recordar la calidad como poetas de dos de los adaptadores: José García Nieto y José Manuel Caballero Bonald. Los dos también, y sobre todo el primero, han versionado obras de nuestro clásico re-

${ }^{16}$ Para las razones de su elección y otros aspectos relacionados con este montaje, puede leerse el coloquio que se celebró en Almagro, después de ser presentada en el Festival. Francisco de Rojas Zorrilla, poeta dramático..., cit., p. 407-411. 
petidamente. Caballero Bonald ya había adaptado para la CNTC Don Gil de las calzas verdes (1994), dirigida por Adolfo Marsillach.

e) Y señalar un tipo de representación, que se repite en diferentes lugares, bien porque los hechos que cuenta la obra se desarrollan en esas ciudades, bien por estar dedicada a personajes históricos o legendarios de las mismas, o por contar los acontecimientos de sus santos patronos o patronas... Este último es el caso de Calahorra, en La Rioja, donde se viene celebrando, desde el año 1999, la puesta en escena (cada mes de agosto un acto) de Los tres blasones de España, en montaje que protagonizan los vecinos y en honor de San Emeterio y San Celedonio.

\section{APÉNDICE}

\section{Refundiciones y adaptaciones en libro}

Abre el ojo, o sea, El aviso a los solteros, comedia en tres actos, escrita por Francisco de Rojas y refundida por D. F. E. Castrillón, 1814 (Al fin= Madrid: lib. de la Viuda de Quiroga).

El amo criado. Comedia de D. Francisco Rojas Zorrilla. Refundida en cinco actos por D. Juan Eugenio Hartzenbusch. Madrid, Yenes, 1841.

Entre bobos anda el juego. Comedia en tres actos de D. Francisco de Rojas; refundida $y$ arreglada en cuatro actos por don Eduardo Asquerino. Madrid, Imp. que fue de Operarios, a cargo de D. F. R. del Castillo, 1851.

Don Lucas del Cigarral. Zarzuela en tres actos y en verso, refundición de la comedia de D. Francisco de Rojas «Entre bobos anda el juego», libro de Tomás Luceño y Carlos Fernández. Shaw; música del maestro Amadeo Vives. Madrid, R. Velasco, 1899.

Amo y criado. Comedia en tres actos y en verso de don Francisco de Rojas, refundida en cuatro por Tomás Luceño. Madrid, Sociedad de autores españoles, 1911.

Lances de amo y criado, comedia lírica en dos actos y en verso. Refundición de la que con el título de «Amo y criado» escribió don Francisco de Rojas. Libro de Tomás Luceño. Música del maestro Rafael Calleja. Madrid, R. Velasco, 1912.

Entre bobos anda el juego. Comedia escrita por el inmortal Francisco de Rojas Zorrilla, arreglada en cuatro actos por L. Suñer Casademunt. Barcelona, F. Costa, 1914.

Amo y criado. Comedia en tres actos, refundida en cuatro por Tomás Luceño. Madrid, Prensa popular («La Novela teatral»), 1923. Es el mismo texto de 1911.

Entre bobos anda el juego. Versión de José García Nieto. Madrid, Teatro Español, 1951. ¡Abre el ojo! Versión de J. M. Caballero Bonald. Madrid, Vox, 1979 (Col. «La Farsa», 1).

Entre bobos anda el juego. Versión de Rafael Pérez Sierra y Gerardo Malla, precedida de una loa de Agustín de Rojas Villandrando. Prólogo de Felipe Pedraza. Madrid, Compañía nacional de teatro clásico [1999]. 